\title{
花后不同时期油菜籽饼粕赖氨酸含量的遗传分析
}

\author{
陈国林 ${ }^{1,2}$ 吴建国 ${ }^{1}$ 张 莉 ${ }^{1}$ 石春海 ${ }^{1, *}$ \\ ${ }^{1}$ 浙江大学农业与生物技术学院, 浙江杭州 $310029 ;{ }^{2}$ 浙江林学院农业与食品学院, 浙江临安 311300
}

摘 要: 采用条件和非条件数量遗传分析方法, 利用不同环境条件下的两年试验数据, 分析了胚(子叶)、细胞质和母 体植株等不同遗传体系的遗传主效应及其环境互作效应对油菜籽饼粕赖氨酸含量的影响。结果表明, 油菜籽饼粕赖 氨酸含量在各个发育时期的表现同时受制于不同遗传体系的遗传主效应和环境互作效应，且以环境互作效应为主， 故油菜籽发育过程中不同遗传体系基因的表达会受到环境条件的显著影响。在不同遗传体系的基因效应中，除花后 $36 \mathrm{~d}$ 的胚主效应较大外, 各发育时期以母体效应为主, 其中母体显性主效应和母体加性互作效应的作用尤为明显。条 件遗传分析表明, 花后 23 29 d 控制赖氨酸含量的数量基因表达最为活跃; 一些基因效应在不同发育时期还存在着 间断性表达的现象。油菜籽饼粕赖氨酸含量的狭义遗传率较高, 多数发育时期是以母体遗传率为主, 根据母体植株上 油菜籽饼粕赖氨酸含量的总体表现进行选择可以取得较好的改良效果。

关键词：油菜籽；赖氨酸含量；发育遗传；遗传主效应；环境互作效应；遗传率

\section{Genetic Analysis for Lysine Content of Rapeseed Cake (Brassic napus L.) at Different Periods after Anthesis}

\author{
CHEN Guo-Lin ${ }^{1,2}$, WU Jian-Guo ${ }^{1}$, ZHANG Li ${ }^{1}$, and SHI Chun-Hai ${ }^{1, *}$ \\ ${ }^{1}$ College of Agriculture and Biotechnology, Zhejiang University, Hangzhou 310029, China; ${ }^{2}$ School of Biosystems Engineering and Food Sciences, \\ Zhejiang Forestry University, Lin'an 311300, China
}

\begin{abstract}
Both conditional and unconditional analysis methods were applied to estimate the genetic main effects and the genotype $\times$ environment $(\mathrm{GE})$ interaction effects from embryo (cotyledon), cytoplasmic and maternal plant at different periods after anthesis for lysine content of rapeseed cake by using two-year experimental data. Lysine content (LC) in rapeseed cake was simultaneously controlled by the genetic main effects and their GE interaction effects from different genetic systems, especially for the GE interaction effects. Therefore, the expression of genes conferring LC in the different genetic systems could be significantly influenced by the environments. Among the different genetic components, the maternal effects on LC of rapeseed cake were the most important at all periods after anthesis except for the larger embryo main effects at 36 day after flowering, in which both maternal dominance effect and maternal additive interaction effect were more visible. It was found that the new expression of quantitative genes on LC trait was most active at 23-29 days after flowering by using the conditional statistical analysis method. The discontinuity of gene expression in the developmental process of rapeseed was also observed. The total narrow-sense heritability of LC was high, of which the maternal heritability was important at most periods after anthesis. It was suggested that LC could be efficiently improved when selection is based on the holistic performance of maternal plants in generations. The study further suggested that Eyouchangjia and Gaoyou 605 were better than other parents for increasing LC of progenies.

Keywords: Rapeseed (Brassica napus L.); Lysine content; Developmental genetics; Genetic main effects; Genotype $\times$ environment interaction effects; Heritability
\end{abstract}

赖氨酸为碱性氨基酸, 是人和动物营养的八种必需 氨基酸之一, 对调节体内代谢平衡、促进生长发育有着重 要作用。油菜是我国大面积种植的油料作物, 油菜籽富含 油脂和蛋白质, 必需氨基酸含量平衡, 优质油菜饼粕是一
种理想蛋白源 ${ }^{[1]}$ 。因此, 提高油菜籽饼粕的必需氨基酸含 量, 尤其是赖氨酸含量对改善饼粕的品质具有重要作用。

氨基酸营养品质在水稻、大麦、小麦等作物已有较 多研究, 其中对赖氨酸 ${ }^{[2-4]}$ 和其他氨基酸 ${ }^{[4-6]}$ 主要采用性状

\section{本研究由浙江省科技厅面上科研农业项目(2008C22084)，教育部高等学校骨干教师资助计划项目和浙江省“151 人才工程”专项基金项目资助。}

*通讯作者(Corresponding author): 石春海, E-mail: chhshi@zju.edu.cn; Tel: 0571-86971691

第一作者联系方式: E-mail: glchen@zju.edu.cn

Received(收稿日期): 2009-09-10; Accepted(接受日期): 2009-12-25. 
的最终表型值进行遗传分析。在油菜籽含油量、脂肪酸、 硫式、芥酸等性状的遗传研究方面也已取得明显的进展。 $\mathrm{Wu}$ 等 ${ }^{[7]}$ 发现胚(子叶)、细胞质和母体植株等遗传体系的 基因主效应及其互作效应可以显著影响油菜籽的蛋白质 含量, 其狭义遗传率达到 $64.17 \%$, 基于母体植株的总体 表现进行蛋白质含量的单株选择可以取得较好的效果。任 玉玲等 ${ }^{[8]}$ 研究结果也表明油菜籽饼粕中谷氨酸、甘氨酸和 精氨酸性状的表达均受到胚、细胞质和母体植株等遗传效 应的控制, 其中谷氨酸和甘氨酸性状的表现是以母体遗 传效应为主, 但精氨酸性状的胚和母体遗传效应相近; 谷 氨酸、甘氨酸和精氨酸的狭义遗传率分别为 $43.4 \%$ 、 $45.6 \%$ 和 $72.1 \%$ 。V Variath 等 ${ }^{[9]}$ 分析了单年份数据后发现控制油菜 籽蛋白质和含油量的胚、细胞质和母体植株基因在各个油 菜籽发育阶段均有表达, 母体植株核基因所表达的遗传 效应在多数发育阶段起着主要作用, 且加性效应要大于 显性效应; 多数发育时段的母体遗传率较大，但尚未明确 环境互作效应对这些性状表现的影响。所以, 研究油菜籽 氨基酸含量的动态遗传效应和发育遗传机理, 对提高品 质有着重要的意义。但至今有关油菜籽饼粕赖氨酸等氨基 酸性状在不同环境条件下的发育遗传研究鲜见报道。本试 验旨在利用甘蓝型油菜品种和组合, 研究不同环境条件 下油菜籽充实过程中赖氨酸含量的发育遗传规律, 明确 油菜籽不同发育时期二倍体胚、细胞质和二倍体母体植株 等不同遗传体系的基因效应差异, 研究结果可为油菜籽 赖氨酸品质的遗传改良提供理论依据。

\section{1 材料与方法}

\section{1 试验材料}

采用油菜籽饼粕赖氨酸含量差异较大的 9 个甘蓝型 油菜品种, 包括油菜 601 $\left(\mathrm{P}_{1}\right)$ 、双 20-4 $\left(\mathrm{P}_{2}\right)$ 、华双 3 号 $\left(\mathrm{P}_{3}\right)$ 、 高油 605 $\left(\mathrm{P}_{4}\right)$ 、中油 $821\left(\mathrm{P}_{5}\right)$ 、鄂油长荚 $\left(\mathrm{P}_{6}\right)$ 、中 $\mathrm{R}-888\left(\mathrm{P}_{7}\right)$ 、 Tower $\left(\mathrm{P}_{8}\right)$ 和浙双 $72\left(\mathrm{P}_{9}\right)$ 。2005 年采用完全双列杂交的方 法配置杂交组合。2006-2007 年和 2007-2008 年分别在 浙江大学实验农场种植亲本和 $F_{1}$, 其中 2006 年为 10 月 9 日播种、2007 年为 10 月 13 日播种, $38 \mathrm{~d}$ 苗龄时移栽。单 株种植, 随机区组设计, 2 次重复。行株距 $35 \mathrm{~cm} \times 30 \mathrm{~cm}$, 各小区种 32 株, 田间管理同一般大田。开花期间在亲本 和 $\mathrm{F}_{1}$ 植株上选择合适花序去除已开花朵, 保留当天开花 花朵, 套袋自交。花后 $15 \mathrm{~d}$ 开始, 每 $7 \mathrm{~d}(15 、 22 、 29 、 36$ 和 $43 \mathrm{~d}$ )收获套袋自交的亲本和 $F_{1}$ 植株上的 $F_{2}$ 种子。在开 花期间利用亲本配制相应的杂交组合, 获得不同发育时 期当代杂交的 $\mathrm{F}_{1}$ 种子。根据油菜籽性状的发育进程, 5 个 发育时段分别称为发育始期(1 15d)、前期 $(16 \sim 22 d)$ 、中 期(23 29 d)、后期(30 36 d)和成熟期(37 43 d)。

\section{2 构建定标方程}

原始样本 621 份油菜籽来自于本课题组 1998、1999、 2003、2005 和 2006 年遗传研究的 5 个不同发育时期, 包 括不同时期的 $F_{1}$ 和 $F_{2}$ 及其亲本，原始样本变异大。各样
品取 $3 \mathrm{~g}$ 油菜籽, 利用美国马里兰州生产的 FOSS NIRSystems 5000 型近红外分析仪，用 $35 \mathrm{~mm}$ 圆杯扫描和收集 样品光谱 ${ }^{[10]}$ 。经 WinISI II(Ver.1.04)近红外分析软件的集 中程序和选择程序筛选样本, 利用具有代表性的 226 份样 品构成校正集，均包括有不同发育时期的油菜籽样品。使 用氨基酸 L-8900 自动分析仪测定去脂后的校正集样品油 菜籽饼粕的氨基酸含量 $(\%)^{[11]}$ 。光谱预处理对校正结果影 响较大, 不同光谱数学处理以 2 阶导数处理较好。最终, 采用 标准正态变量转换(SNV)+趋势变换法(De-trending)/ 2,4,4,1/MPLS” 最优组合构建油菜籽饼粕氨基酸含量的定 标方程, 其中油菜籽饼粕赖氨酸含量定标方程决定系数 (RSQ)达到 0.972 , 标准误(SEC)为 $0.116 \%$ 。

\section{3 赖氨酸含量测定}

利用上述新建立的定标方程 $(\mathrm{RSQ}=0.972, \mathrm{SEC}=$ $0.116 \%$ ), 在 FOSS NIRSystems 5000 型近红外分析仪上测 定和分析两年亲本、 $F_{1}$ 和 $F_{2}$ 油菜籽 5 个不同发育时段饼 粕的赖氨酸含量。扫描时取 $3 \mathrm{~g}$ 油菜籽, 用 $35 \mathrm{~mm}$ 圆杯收 集样品光谱。亲本、 $F_{1}$ 和 $F_{2}$ 样本的测定各重复 2 次。

1.4 统计分析

采用包括胚、细胞质和母体植株等不同遗传体系基 因主效应和环境互作效应的非条件分析方法 ${ }^{[12]}$,估算油 菜开花受精至不同发育时段 $(0 \rightarrow t)$ 控制赖氨酸含量性状表 现的累积遗传效应方差值 $\left(V_{G t(t)}\right)$, 包括胚加性 $\left(V_{A(t)}\right)$ 和显 性 $\left(V_{D(t)}\right)$ 方差、细胞质方差 $\left(V_{C(t)}\right)$ 、母体加性 $\left(V_{A m(t)}\right)$ 和显性 $\left(V_{D m(t)}\right)$ 方差、胚加性互作 $\left(V_{A E(t)}\right)$ 和显性互作 $\left(V_{D E(t)}\right)$ 方差、 细胞质互作方差 $\left(V_{C E(t)}\right)$ 、母体加性互作 $\left(V_{A m E(t)}\right)$ 和显性互作 $\left(V_{D m E(t)}\right)$ 方差、剩余方差 $\left(V_{e(t)}\right)$ 等方差分量以及胚加性与母 体加性效应间协方差 $\left(C_{A \cdot A m(t)}\right)$ 、胚显性与母体显性效应间 协方差 $\left(C_{D \cdot D m(t)}\right)$ 、胚加性互作与母体加性互作效应间协方 差 $\left(C_{A E \cdot A m E(t)}\right)$ 、胚显性互作与母体显性互作效应间协方差 $\left(C_{D E \cdot D m E(t)}\right)$ 。同时采用发育遗传模型和条件分析方法估算 油菜籽某一特定发育时期 $((t-1) \rightarrow t)$ 基因新表达的净遗传 效应方差值 $\left(V_{G(t \mid t-1)}\right)^{[13]}$, 包括条件胚加性 $\left(V_{A(t \mid t-1)}\right)$ 和显性 $\left(V_{D(t \mid t-1)}\right)$ 方差、条件细胞质方差 $\left(V_{C(t \mid t-1)}\right)$ 、条件母体加性 $\left(V_{A m(t \mid t-1)}\right)$ 和显性 $\left(V_{D m(t \mid t-1)}\right)$ 方差、条件胚加性互作 $\left(V_{A E(t \mid t-1)}\right)$ 和显性互作 $\left(V_{D m E(t \mid t-1)}\right)$ 方差、条件细胞质互作方差 $\left(V_{C E(t \mid t-1)}\right)$ 、 条件母体加性互作 $\left(V_{A m E(t|t|-1)}\right)$ 和显性互作 $\left(V_{D m E(t \mid t-1)}\right)$ 方差、条 件剩余方差 $\left(V_{e(t) \mid-1)}\right)$ 以及条件胚加性与条件母体加性效应 间协方差 $\left(C_{A \cdot A m(t \mid t-1)}\right)$ 、条件胚显性与条件母体显性间协方 差 $\left(C_{D \cdot D m(t \mid t-1)}\right)$ 、条件胚加性互作效应与条件母体加性互作 效应间协方差 $\left(C_{A E \cdot A m E(t \mid t-1)}\right)$ ，条件胚显性互作效应与条件 母体显性互作效应间协方差 $\left(C_{D E \cdot D m E(t \mid t-1)}\right)$, 其中 $(t \mid t-1)$ 是 指给定第 $(t-1)$ 个油菜籽发育时期赖氨酸含量时第 $t$ 个发育 时期的条件表型值。根据遗传方差分解原理，总狭义遗传 率 $\left(h^{2}\right)$ 可以进一步分解为胚普通遗传率 $\left(h_{G o}^{2}\right)$ 、细胞质普通 遗传率 $\left(h_{G c}^{2}\right)$ 、母体普通遗传率 $\left(h_{G m}^{2}\right)$ 、胚互作遗传率 $\left(h_{G o E}^{2}\right)$ 、细胞质互作遗传率 $\left(h_{G c E}^{2}\right)$ 和母体互作遗传率 
$\left(h_{G m E}^{2}\right)$ 等各项遗传率分量。采用 AUP 法预测胚加性效应 $(A)$ 、细胞质效应 $(C)$ 、母体加性效应 $(A m)$ 、胚加性互作效 应 $(A E)$ 、细胞质互作效应 $(C E)$ 和母体加性互作效应 $(A m E)$ 等各项遗传效应分量。采用 Jackknife 抽样技术对各世代 平均数进行抽样, 计算方差、协方差、遗传率、亲本效应 预测值等各项参数估计值的标准误。

\section{2 结果与分析}

2.1 籽粒不同发育时段亲本及杂交后代赖氨酸含量的平 均表现

2006 年亲本和杂种后代 $\left(F_{1}\right.$ 和 $\left.F_{2}\right)$ 在开花后 $15 、 22$ 、

29、36 和 $43 \mathrm{~d}$ 时的油菜籽饼粕赖氨酸含量平均值, 分别
为 $0.683 \% 、 1.197 \% 、 1.478 \% 、 2.047 \%$ 和 $2.061 \%$ ，相应的 标准差为 $0.219 \% 、 0.531 \% 、 0.384 \% 、 0.390 \%$ 和 $0.344 \%$ 。 $F_{1}$ 分别为 $0.951 \% 、 1.212 \% 、 1.497 \% 、 1.927 \%$ 和 $2.265 \%$, 其 标准差为 $0.226 \% 、 0.598 \% 、 0.514 \% 、 0.283 \%$ 和 $0.240 \%$ ，表 现出明显的杂种优势。 $F_{2}$ 与亲本相近, 为 $0.727 \% \sim 2.077 \%$, 相应的标准差为 $0.196 \%$ 0.356\% (表 1)。2007 年与 2006 年的结果有着较大差异, 亲本和杂交后代在不同发育时 段的表现型值差异较大, 说明赖氨酸含量在不同发育时 段表现动态变化的过程, 随着发育时期的延续其赖氨酸 含量明显增加, 以成熟时最大。上述结果表明供试材料和 各个发育时期的赖氨酸含量存在着明显差异, 环境条件 也有着明显的影响。

表 1 油菜籽饼粕中赖氨酸含量平均值和变异范围

Table 1 Means and ranges for lysine content in rapeseed cake

\begin{tabular}{|c|c|c|c|c|c|c|c|c|c|}
\hline \multirow{3}{*}{$\begin{array}{c}\text { 世代 } \\
\text { Generation }\end{array}$} & \multirow{3}{*}{$\begin{array}{c}\text { 发育时段 } \\
\text { Growth } \\
\text { period (d) }\end{array}$} & \multicolumn{4}{|c|}{2006} & \multicolumn{4}{|c|}{2007} \\
\hline & & \multirow{2}{*}{$\begin{array}{l}\text { 平均值 } \\
\text { Mean }\end{array}$} & \multicolumn{2}{|c|}{ 变异范围 } & \multirow{2}{*}{$\begin{array}{c}\text { 标准差 } \\
S D\end{array}$} & \multirow{2}{*}{$\begin{array}{c}\text { 平均值 } \\
\text { Mean }\end{array}$} & \multicolumn{2}{|c|}{ 变异范围 } & \multirow{2}{*}{$\begin{array}{c}\text { 标准差 } \\
S D\end{array}$} \\
\hline & & & Min. & Max. & & & Min. & Max. & \\
\hline \multirow{5}{*}{$\begin{array}{l}\text { 亲本 } \\
\text { Parent }\end{array}$} & 15 & 0.683 & 0.317 & 1.092 & 0.219 & 1.310 & 0.657 & 2.031 & 0.370 \\
\hline & 22 & 1.197 & 0.570 & 2.126 & 0.531 & 0.712 & 0.000 & 1.235 & 0.442 \\
\hline & 29 & 1.478 & 0.941 & 2.014 & 0.384 & 1.252 & 0.452 & 2.195 & 0.486 \\
\hline & 36 & 2.047 & 1.268 & 2.627 & 0.390 & 1.857 & 1.209 & 2.555 & 0.430 \\
\hline & 43 & 2.061 & 1.453 & 2.480 & 0.344 & 2.196 & 1.868 & 2.499 & 0.182 \\
\hline \multirow[t]{5}{*}{$\mathrm{F}_{1}$} & 15 & 0.951 & 0.489 & 1.445 & 0.226 & 0.933 & 0.390 & 1.810 & 0.318 \\
\hline & 22 & 1.212 & 0.564 & 2.469 & 0.598 & 0.323 & 0.000 & 1.168 & 0.316 \\
\hline & 29 & 1.497 & 0.601 & 2.492 & 0.514 & 1.471 & 0.274 & 2.403 & 0.654 \\
\hline & 36 & 1.927 & 0.993 & 2.406 & 0.283 & 2.099 & 1.408 & 2.483 & 0.254 \\
\hline & 43 & 2.265 & 1.668 & 2.750 & 0.240 & 2.275 & 1.751 & 2.620 & 0.188 \\
\hline \multirow[t]{5}{*}{$\mathrm{F}_{2}$} & 15 & 0.727 & 0.355 & 1.375 & 0.196 & 1.001 & 0.543 & 1.752 & 0.203 \\
\hline & 22 & 1.136 & 0.545 & 2.136 & 0.356 & 0.796 & 0.000 & 1.661 & 0.420 \\
\hline & 29 & 1.464 & 0.794 & 2.392 & 0.292 & 0.945 & 0.349 & 2.052 & 0.339 \\
\hline & 36 & 1.837 & 1.349 & 2.404 & 0.240 & 1.902 & 1.286 & 2.452 & 0.271 \\
\hline & 43 & 2.077 & 1.521 & 2.640 & 0.269 & 2.105 & 1.436 & 2.437 & 0.173 \\
\hline
\end{tabular}

2.2 不同发育时段赖氨酸含量的遗传分析

非条件遗传方差分量结果表明, 油菜籽不同发育时 段的饼粕赖氨酸含量同时受制于胚、细胞质和母体植株等 不同遗传体系的基因主效应和环境互作效应(表 2)。遗传 主效应方差 $\left(V_{G(t)}=V_{A(t)}+V_{D(t)}+V_{C(t)}+V_{A m(t)}+V_{D m(t)}\right)$ 和环 境互作效应方差 $\left(V_{G E(t)}=V_{A E(t)}+V_{D E(t)}+V_{C E(t)}+V_{A m E(t)}+\right.$ $\left.V_{D m E(t)}\right)$ 的分析表明，花后 $15 、 22 、 29 、 36$ 和 $43 \mathrm{~d}$ 的赖氨 酸含量均以环境互作效应为主, 分别占各时段遗传方差 总量 $\left(V_{G t(t)}\right)$ 的 $82.48 \% 、 87.54 \% 、 89.29 \% 、 60.95 \%$ 和 $71.80 \%$, 说明控制油菜籽饼粕赖氨酸含量的基因在不同发育时段 的表达受环境条件的影响较大。

在遗传主效应中, 花后 $36 \mathrm{~d}$ 可以检测到胚加性主效 应方差和细胞质主效应方差, 而胚显性主效应方差除了 $29 \mathrm{~d}$ 外均达极显著水平。虽然仅在花后 $43 \mathrm{~d}$ 能够测到极
显著的母体加性主效应方差，但不同发育时段的母体显 性主效应方差均已达极显著水平。因此，控制油菜籽饼粕 赖氨酸含量性状的基因表达在不同发育时段有着明显差 异, 其中以二倍体母体植株核基因产生的母体显性主效 应尤为明显, 其次为二倍体胚核基因产生的胚显性主效 应。在环境互作效应中, 除 $36 \mathrm{~d}$ 末检测到细胞质互作方差 $\left(V_{C E(36)}\right)$ 外, 各时段环境互作方差分量 $\left(V_{A E(t) 、} 、 V_{D E(t) 、} 、 V_{C E(t)}\right.$ 、 $\left.V_{A m E(t) 、} V_{D m E(t)}\right)$ 均达极显著水平。而且各发育时段的母体 互作方差分别占环境互作方差总量 $\left[\left(V_{A m E(t)}+V_{D m E(t)}\right) / V_{G E(t)}\right]$ 的 $47.21 \% 、 50.86 \% 、 50.31 \% 、 54.53 \%$ 和 $45.96 \%$ ，表明这 些发育时段均以母体互作效应为主。表 2 结果还表明细胞 质互作效应对油菜籽不同发育时段赖氨酸含量也起着较 为明显作用。

由于胚与母体间的遗传主效应协方差 $\left(C_{A \cdot A m(t)}\right.$ 、 
表 2 油菜籽饼粕赖氨酸含量在不同发育时段的方差估算值

Table 2 Estimates of variance for lysine content at different development periods in rapeseed cake

\begin{tabular}{|c|c|c|c|c|c|}
\hline \multirow{3}{*}{$\begin{array}{c}\text { 参数 } \\
\text { Parameter }\end{array}$} & \multicolumn{5}{|c|}{ 非条件分析 Uncondition analysis } \\
\hline & \multicolumn{5}{|c|}{ 油菜籽发育时段 Developmental period of rapeseed } \\
\hline & $15 \mathrm{~d}$ & $22 \mathrm{~d}$ & $29 \mathrm{~d}$ & $36 \mathrm{~d}$ & $43 \mathrm{~d}$ \\
\hline$V_{A(t)}$ & 0.000 & 0.000 & 0.000 & $0.087^{* *}$ & 0.000 \\
\hline$V_{D(t)}$ & $0.031^{* *}$ & $0.061^{* *}$ & 0.000 & $0.022^{* *}$ & $0.010^{* *}$ \\
\hline$V_{C(t)}$ & 0.000 & 0.000 & 0.000 & $0.020^{* *}$ & 0.000 \\
\hline$V_{A m(t)}$ & 0.000 & 0.000 & 0.000 & 0.000 & $0.042^{* *}$ \\
\hline$V_{D m(t)}$ & $0.060^{* *}$ & $0.135^{* *}$ & $0.185^{* *}$ & $0.035^{* *}$ & $0.023^{* *}$ \\
\hline$V_{A E(t)}$ & $0.097^{* *}$ & $0.434^{* *}$ & $0.463^{* *}$ & $0.081^{* *}$ & $0.045^{* *}$ \\
\hline$V_{D E(t)}$ & $0.035^{* *}$ & $0.052^{* *}$ & $0.194^{* *}$ & $0.035^{* *}$ & $0.011^{* *}$ \\
\hline$V_{C E(t)}$ & $0.094^{* *}$ & $0.190^{* *}$ & $0.108^{* *}$ & 0.000 & $0.047^{* *}$ \\
\hline$V_{A m E(t)}$ & $0.134^{* *}$ & $0.557^{* *}$ & $0.494^{* *}$ & $0.100^{* *}$ & $0.056^{* *}$ \\
\hline$V_{D m E(t)}$ & $0.068^{* *}$ & $0.142^{* *}$ & $0.280^{* *}$ & $0.040^{* *}$ & $0.032^{* *}$ \\
\hline$C_{A \cdot A m(t)}$ & 0.000 & 0.000 & 0.000 & 0.000 & 0.000 \\
\hline$C_{D \cdot D m(t)}$ & -0.011 & -0.017 & 0.000 & -0.007 & 0.000 \\
\hline$C_{A E: A m E(t)}$ & -0.067 & -0.198 & -0.017 & -0.039 & -0.002 \\
\hline$C_{D E \cdot D m E(t)}$ & -0.002 & 0.000 & -0.076 & 0.004 & -0.003 \\
\hline$V e_{(t)}$ & $0.025^{* *}$ & $0.088^{* *}$ & $0.045^{* *}$ & $0.028^{* *}$ & $0.022^{* *}$ \\
\hline \multirow{3}{*}{$\begin{array}{c}\text { 参数 } \\
\text { Parameter }\end{array}$} & \multicolumn{5}{|c|}{ 条件分析 Condition analysis } \\
\hline & \multicolumn{5}{|c|}{ 油菜䊏发育时段 Developmental period of rapeseed } \\
\hline & $5 \mathrm{~d} \mid 0 \mathrm{~d}$ & $22 \mathrm{~d} \mid 15 \mathrm{~d}$ & $29 \mathrm{~d} \mid 22 \mathrm{~d}$ & $36 \mathrm{~d} \mid 29 \mathrm{~d}$ & $43 \mathrm{~d} \mid 36 \mathrm{~d}$ \\
\hline$V_{A(t \mid t-1)}$ & 0.000 & 0.000 & 0.000 & $0.072^{* *}$ & 0.000 \\
\hline$V_{D(t \mid t-1)}$ & $0.031^{* *}$ & $0.058^{* *}$ & $0.337^{* *}$ & 0.000 & $0.010^{* *}$ \\
\hline$V_{C(t \mid t-1)}$ & 0.000 & 0.000 & 0.000 & $0.011^{* *}$ & 0.000 \\
\hline$V_{A m(t \mid t-1)}$ & 0.000 & 0.000 & 0.000 & 0.000 & 0.000 \\
\hline$V_{D m(t \mid t-1)}$ & $0.060^{* *}$ & $0.135^{* *}$ & $0.368^{* *}$ & $0.031^{* *}$ & $0.017^{* *}$ \\
\hline$V_{A E(t \mid t-1)}$ & $0.097^{* *}$ & $0.373^{* *}$ & $1.395^{* *}$ & $0.081^{* *}$ & $0.040^{* *}$ \\
\hline$V_{D E(t \mid t-1)}$ & $0.035^{* *}$ & $0.051^{* *}$ & $0.360^{* *}$ & $0.037^{* *}$ & $0.010^{* *}$ \\
\hline$V_{C E(t \mid t-1)}$ & $0.094^{* *}$ & $0.185^{* *}$ & $0.777^{* *}$ & $0.041^{* *}$ & $0.050^{* *}$ \\
\hline$V_{A m E(t \mid t-1)}$ & $0.134^{* *}$ & $0.479^{* *}$ & $6.499^{* *}$ & $0.094^{* *}$ & $0.057^{* *}$ \\
\hline$V_{D m E(t \mid t-1)}$ & $0.068^{* *}$ & $0.150^{* *}$ & $0.572^{* *}$ & $0.039^{* *}$ & $0.031^{* *}$ \\
\hline$C_{A \cdot A m(t \mid t-1)}$ & 0.000 & 0.000 & 0.000 & 0.000 & 0.000 \\
\hline$C_{D \cdot D m(t \mid t-1)}$ & -0.011 & -0.014 & -0.120 & 0.000 & -0.002 \\
\hline$C_{A E: A m E(t \mid t-1)}$ & -0.067 & -0.107 & -0.828 & -0.043 & 0.000 \\
\hline$C_{D E \cdot D m E(t \mid t-1)}$ & -0.002 & -0.004 & -0.086 & 0.004 & -0.003 \\
\hline$V_{e(t \mid t-1)}$ & $0.025^{* *}$ & $0.087^{* *}$ & $0.053^{* *}$ & $0.027^{* *}$ & $0.021^{* *}$ \\
\hline
\end{tabular}

"*表示达到 0.01 显著水平。"* Significant at 0.01 probability level.

$V_{A(t)}$ : embryo additive variance; $V_{D(t)}$ : embryo dominance variance; $V_{C(t)}$ : cytoplasmic variance; $V_{A m(t)}$ : maternal additive variance; $V_{D m(t)}$ : maternal dominance variance; $V_{A E(t)}$ : embryo additive interaction variance; $V_{D E(t)}$ : embryo dominance interaction variance; $V_{C E(t)}$ : cytoplasmic interaction variance; $V_{A m E(t)}$ : maternal additive interaction variance; $V_{D m E(t)}$ : maternal dominance interaction variance; $C_{A \cdot A m(t)}$ : additive covariance; $C_{D \cdot D m(t)}:$ dominant covariance; $C_{A E \cdot A m E(t)}$ : additive interaction covariance; $C_{D E \cdot D m E(t)}$ : dominant interaction covariance; $V_{e(t)}$ : residual variance. $V_{A(t \mid t-1)}$ : conditional embryo additive variance; $V_{D(t \mid t-1)}$ : conditional embryo dominance variance; $V_{C(t \mid t-1)}$ : conditional cytoplasmic variance; $V_{A m(t \mid t-1)}$ : conditional maternal additive variance; $V_{D m(t \mid t-1)}$ : conditional maternal dominance variance; $V_{A E(t \mid t-1)}$ : conditional embryo additive interaction variance; $V_{D E(t \mid t-1)}$ : conditional embryo dominance interaction variance; $C_{A \cdot A m(t \mid t-1)}$ : conditional additive covariance; $C_{D \cdot D m(t \mid t-1)}$ : conditional dominance covariance; $C_{A E \cdot A m E(t \mid t-1)}$ : conditional additive interaction covariance; $C_{D E \cdot D m E(t \mid t-1)}$ : conditional dominance interaction covariance; $V_{e(t \mid t-1)}$ : conditional residual variance.

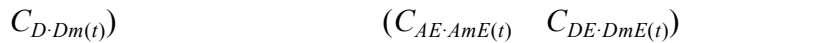
著水平，因此不同发育时段二倍体胚效应对赖氨酸含量 的影响与母体效应没有明显的关系。虽然油菜籽各发育时 段的机误方差 $\left(V_{e(t)}\right)$ 均达极显著水平, 环境机误或抽样误 差对油菜籽饼粕赖氨酸含量的表现有一定影响, 但由于 其值相对较小, 该性状的表现主要受制于基因主效应和

\section{环境互作效应。}

\section{3 不同发育时期赖氨酸含量的遗传分析}

采用非条件分析方法获得的油菜籽不同发育时段 $(0$ $\rightarrow t$ )遗传方差仅能反映出油菜开花至 $t$ 时段基因表达产生 遗传效应的累加值, 不能反映出其中某一段特定发育时 期 $(t-1 \rightarrow t)$ 基因表达的情况。而条件方差分析的方法则能 
够获得在某一特定发育时期中控制赖氨酸含量的基因新 表达的净遗传效应, 可有效解释油菜籽不同发育时期各 种遗传效应的动态变化。条件方差分量分析结果表明, 胚、细胞质或母体植株中控制赖氨酸含量性状表现的基因 在油菜籽多数发育时期均有新的表达(表 2)。5 个油菜籽发 育时期中基因新表达的条件遗传主效应方差分别占遗传 总方差值的 $17.52 \% 、 13.47 \% 、 6.84 \% 、 28.00 \%$ 和 $12.36 \%$ $\left(V_{G(t \mid t-1)} / V_{G t(t t-1)}\right)$, 而条件互作效应方差分别占 $82.48 \%$ 、 $86.53 \% 、 93.16 \% 、 72.00 \%$ 和 $87.64 \%\left(V_{G E(t \mid t-1)} / V_{G t(t \mid t-1)}\right)$, 说 明各发育时期新表达的净遗传效应是以条件互作效应为 主, 其基因表达容易受到环境条件变化的影响, 特别是对 发育中期基因表达的影响尤为明显。条件互作遗传效应中 是以条件母体互作效应为主, 分别占互作方差总值的 $47.21 \% 、 50.81 \% 、 73.64 \% 、 45.50 \%$ 和 $46.80 \%\left(V_{A m E(t \mid t-1)}{ }^{+}\right.$ $\left.V_{D m E(t \mid t-1)} / V_{G E(t \mid t-1)}\right)$; 其次为条件胚互作效应, 分别占 $30.76 \%$ 、 $34.25 \% 、 18.27 \%$ 、 $40.39 \%$ 和 $26.62 \%\left(V_{A E(t \mid t-1)}+V_{D E(t \mid t-1)} /\right.$ $\left.V_{G E(t \mid t-1)}\right)$ 。因此, 就总体而言, 各发育时期新表达的基因 效应是以条件母体主效应为主, 其中又以条件母体显性 主效应尤为明显。由于不同发育时期的净细胞质互作效应 方差均已达极显著水平, 细胞质基因在各个发育时期的 表达也不能忽视。

在油菜籽各个发育时期中, 中期(开花受精后 22 29 d) 是控制赖氨酸含量表现的数量基因表达最为明显的时 期, 控制油菜赖氨酸含量表现的净遗传效应要明显大于 其他发育时期。上述结果表明油菜籽发育中期的基因表达 量为最大, 说明这一特定时期有大量的微效多基因被激 活表达。另外，发育前期基因表达的净遗传效应也较大。 条件分析的结果还表明，一些基因的遗传效应存在着不 同发育时期间断表达的现象，如胚显性主效应有关的基 因在前三期均有新的表达，但在发育后期(30 36 d)未检 测到净效应，成熟期(37 43 d)又重新表达; 胚加性主效应
和细胞质主效应的基因也仅在发育后期(30 36 d)表达。另 外，在花后 23 29 d 期间利用条件方差分析法可以检测到 净胚显性主效应，但在非条件方差分析时的花后 $29 \mathrm{~d}$ 则 未检测到胚显性主效应, 说明条件分析法比非条件法可 以更早检测到新表达的基因效应，非条件方差分析中在 花后 $36 \mathrm{~d}$ 检测到的胚显性主效应是相关基因前期表达而 产生的后续遗传效应。

遗传主效应条件协方差 $\left(C_{A \cdot A m(t \mid t-1) 、} C_{D \cdot D m(t \mid t-1)}\right)$ 及条件互

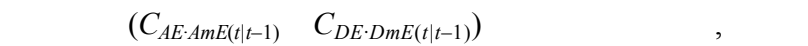
不同发育期间净胚效应对油菜赖氨酸含量的影响与净母 体效应也不存在明显关系。

\section{4 赖氨酸含量在不同发育时段的遗传率分析}

由于遗传方差可按变异来源分解成属于不同遗传体 系的各种方差分量, 遗传率也可作相应分解, 能够更清晰 地反映出各遗传体系基因效应在性状遗传中的相对重要 性。各项遗传率分量中除了花后 $36 \mathrm{~d}$ 是以普通遗传率为 主 $(29.6 \%)$ 外, 其他发育时段的遗传率以互作遗传率为主, 分别为 $49.4 \%$ 、 $64.0 \%$ 、 $65.0 \%$ 和 $52.0 \%$ ，表明油菜籽发育 过程赖氨酸含量的遗传率受环境影响较大(表 3)。前 3 个 时段未检测到普通遗传率分量, 仅在花后 $36 \mathrm{~d}$ 检测到极 显著的胚普通遗传率 $\left(h_{G o}^{2}=0.241^{* *}\right)$ 和细胞质普通遗传率 $\left(h_{G c}^{2}=0.055^{* *}\right)$ 以及花后 $43 \mathrm{~d}$ 极显著的母体普通遗传率 $\left(h_{G m}^{2}=0.153^{* *}\right)$ 。在互作遗传率上, 除花后 $36 \mathrm{~d}$ 未检测到 细胞质互作遗传率 $\left(h_{G c E}^{2}\right)$ 外, 其他互作遗传率分量达显 著或极显著水平。在不同遗传体系中, 除花后 $36 \mathrm{~d}$ 是以胚 遗传率为主外, 其他发育时段是以母体遗传率加细胞质 遗传率为主，分别占狭义遗传率的 $84.5 \%$ 、69.9\%、56.8\% 和 $76.7 \%$ 。因此，根据母体植株的油菜籽饼粕赖氨酸含量 的总体表现在各世代进行选择, 有利于提高赖氨酸含量 的育种效率。

表 3 不同发育时段油菜籽饼粕赖氨酸含量遗传率估计值

Table 3 Estimates of heritability at different development periods for lysine content of rapeseed cake

\begin{tabular}{ccccc}
\hline $\begin{array}{c}\text { 参数 } \\
\text { Parameter }\end{array}$ & \multicolumn{3}{c}{ 油菜籽发育时段 } & Developmental periods of rapeseed \\
\cline { 2 - 4 }$h_{G o}^{2}$ & $15 \mathrm{~d}$ & $22 \mathrm{~d}$ & $29 \mathrm{~d}$ & $0.241^{* *}$ \\
$h_{G c}^{2}$ & 0.000 & 0.000 & 0.000 & $0.055^{* *}$ \\
$h_{G m}^{2}$ & 0.000 & 0.000 & 0.000 & 0.000 \\
$h_{G o E}^{2}$ & 0.000 & 0.000 & 0.000 & 0.000 \\
$h_{G c E}^{2}$ & $0.077^{+}$ & $0.192^{*}$ & $0.281^{* *}$ & $0.117^{*}$ \\
$h_{G m E}^{2}$ & $0.245^{* *}$ & $0.154^{* *}$ & $0.068^{* *}$ & 0.000 \\
$0.157^{* *}$ & $0.168^{* *}$ & $0.167^{* *}$ & $0.195^{*}$ \\
\hline
\end{tabular}

、 ${ }^{*}$ 和 ${ }^{* *}$ 分别表示达到 $0.10 、 0.05$ 和 0.01 显著水平。 ${ }^{+},{ }^{*}$, and ${ }^{* *}$ : significantly different at $0.10,0.05$, and 0.01 probability levels, respectively. $h_{G o}^{2}$ : embryo general heritability; $h_{G c}^{2}$ : cytoplasmic general heritability; $h_{G m}^{2}:$ maternal general heritability; $h_{G o E}^{2}:$ embryo interaction heritability; $h_{G c E}^{2}$ : cytoplasmic interaction heritability; $h_{G m E}^{2}:$ maternal interaction heritability.

2.5 亲本在不同发育时段的赖氨酸含量遗传效应分析 亲本遗传效应的分析可以明确各亲本在油菜籽不同发 育阶段育种值的动态表现, 有利于篮选出不同发育时期表
现较好的最佳亲本用于品质改良。亲本预测值表明各亲本的 遗传效应在油菜籽不同发育时期存在着显著的差异(表 4)。 其中有些亲本受环境影响较小，基因在不同环境下的表达 
表 4 油菜籽饼粕赖氨酸含量的亲本遗传效应预测值

Table 4 Predicating parent genetic effects of lysine content in rapeseed cake (\%)

\begin{tabular}{|c|c|c|c|c|c|c|c|c|c|c|c|c|}
\hline \multirow{2}{*}{$\begin{array}{l}\text { 亲本 } \\
\text { Parent }\end{array}$} & \multirow{2}{*}{$\begin{array}{l}\text { 时段 } \\
\text { Period }\end{array}$} & \multicolumn{3}{|c|}{$\begin{array}{c}\text { 胚加性效应 } \\
\text { Embryo additive effect }\end{array}$} & \multicolumn{3}{|c|}{$\begin{array}{c}\text { 细胞质效应 } \\
\text { Cytoplasmic effect }\end{array}$} & \multicolumn{3}{|c|}{$\begin{array}{c}\text { 母体加性效应 } \\
\text { Maternal additive effect }\end{array}$} & \multirow[t]{2}{*}{ Gt1 } & \multirow[t]{2}{*}{ Gt2 } \\
\hline & & $A$ & $A E 1$ & $A E 2$ & $C$ & $C E 1$ & $C E 2$ & $A m$ & $A m E 1$ & $A m E 2$ & & \\
\hline \multirow[t]{5}{*}{$\mathrm{P}_{1}$} & $15 \mathrm{~d}$ & 0.00 & $0.03^{*}$ & $0.30^{*}$ & 0.00 & $0.35^{*}$ & $-0.45^{*}$ & 0.00 & $0.07^{+}$ & $-0.12^{+}$ & 0.45 & -0.27 \\
\hline & $22 \mathrm{~d}$ & 0.00 & 0.02 & 0.09 & 0.00 & $-0.40^{* *}$ & $0.42^{* *}$ & 0.00 & 0.17 & $-0.22^{*}$ & -0.21 & 0.29 \\
\hline & $29 \mathrm{~d}$ & 0.00 & 0.50 & $-0.76^{+}$ & 0.00 & $-0.43^{*}$ & 0.13 & 0.00 & -0.20 & 0.43 & -0.12 & -0.19 \\
\hline & $36 \mathrm{~d}$ & 0.06 & 0.09 & -0.02 & -0.29 & 0.00 & 0.00 & 0.00 & 0.02 & 0.07 & -0.13 & -0.18 \\
\hline & $43 \mathrm{~d}$ & 0.00 & $-0.22^{*}$ & $0.12^{*}$ & 0.00 & $0.19^{*}$ & $-0.19^{*}$ & $0.12^{*}$ & $0.10^{*}$ & $0.11^{*}$ & 0.20 & 0.16 \\
\hline \multirow[t]{5}{*}{$\mathrm{P}_{2}$} & $15 \mathrm{~d}$ & 0.00 & $-0.56^{*}$ & 0.39 & 0.00 & $0.40^{*}$ & $-0.44^{*}$ & 0.00 & 0.11 & -0.08 & -0.04 & -0.13 \\
\hline & $22 \mathrm{~d}$ & 0.00 & $-0.44^{*}$ & $0.31^{*}$ & 0.00 & $0.59^{* *}$ & $-0.74^{* *}$ & 0.00 & $0.26^{+}$ & -0.10 & 0.40 & -0.53 \\
\hline & $29 \mathrm{~d}$ & 0.00 & -0.05 & -0.19 & 0.00 & $0.29^{*}$ & $-0.45^{*}$ & 0.00 & -0.18 & 0.09 & 0.06 & -0.54 \\
\hline & $36 \mathrm{~d}$ & -0.19 & 0.07 & -0.36 & 0.12 & 0.00 & 0.00 & 0.00 & -0.14 & 0.11 & -0.14 & -0.32 \\
\hline & $43 \mathrm{~d}$ & 0.00 & $0.11^{+}$ & -0.06 & 0.00 & $-0.28^{* *}$ & $0.19^{*}$ & $-0.07^{+}$ & -0.06 & -0.04 & -0.29 & 0.02 \\
\hline \multirow[t]{5}{*}{$\mathrm{P}_{3}$} & $15 \mathrm{~d}$ & 0.00 & -0.09 & 0.02 & 0.00 & $0.23^{+}$ & -0.22 & 0.00 & 0.13 & -0.10 & 0.26 & -0.31 \\
\hline & $22 \mathrm{~d}$ & 0.00 & $-0.39^{*}$ & $0.27^{+}$ & 0.00 & 0.00 & 0.05 & 0.00 & $0.35^{+}$ & $-0.24^{+}$ & -0.04 & 0.08 \\
\hline & $29 \mathrm{~d}$ & 0.00 & 0.70 & -0.45 & 0.00 & $0.38^{* *}$ & $-0.62^{* *}$ & 0.00 & -0.54 & 0.47 & 0.54 & -0.60 \\
\hline & $36 \mathrm{~d}$ & $0.21^{*}$ & -0.15 & $0.41^{+}$ & 0.16 & 0.00 & 0.00 & 0.00 & $0.28^{* *}$ & $-0.34^{* *}$ & 0.50 & 0.44 \\
\hline & $43 \mathrm{~d}$ & 0.00 & 0.08 & -0.09 & 0.00 & 0.00 & 0.06 & 0.02 & 0.07 & -0.01 & 0.17 & -0.02 \\
\hline \multirow[t]{5}{*}{$\mathrm{P}_{4}$} & $15 \mathrm{~d}$ & 0.00 & $-0.25^{+}$ & $0.25^{+}$ & 0.00 & $-0.26^{+}$ & -0.11 & 0.00 & $0.28^{*}$ & -0.21 & -0.23 & -0.06 \\
\hline & $22 \mathrm{~d}$ & 0.00 & $-0.36^{+}$ & $0.32^{+}$ & 0.00 & -0.28 & 0.23 & 0.00 & $0.42^{*}$ & $-0.46^{* *}$ & -0.22 & 0.08 \\
\hline & $29 d$ & 0.00 & -0.27 & 0.64 & 0.00 & $0.60^{+}$ & -0.67 & 0.00 & 0.25 & -0.29 & 0.58 & -0.32 \\
\hline & $36 \mathrm{~d}$ & $0.12^{+}$ & 0.05 & 0.11 & -0.09 & 0.00 & 0.00 & 0.00 & -0.05 & 0.10 & 0.02 & 0.23 \\
\hline & $43 \mathrm{~d}$ & 0.00 & 0.11 & 0.03 & 0.00 & 0.08 & $-0.12^{+}$ & 0.02 & -0.03 & $0.08^{+}$ & 0.18 & 0.02 \\
\hline \multirow[t]{5}{*}{$\mathrm{P}_{5}$} & $15 \mathrm{~d}$ & 0.00 & -0.06 & $-0.24^{+}$ & 0.00 & 0.03 & -0.20 & 0.00 & 0.06 & 0.05 & 0.02 & -0.39 \\
\hline & $22 \mathrm{~d}$ & 0.00 & -0.14 & 0.09 & 0.00 & $-0.55^{*}$ & $0.69^{*}$ & 0.00 & 0.33 & -0.27 & -0.36 & 0.51 \\
\hline & $29 \mathrm{~d}$ & 0.00 & 0.41 & -0.39 & 0.00 & -0.24 & 0.21 & 0.00 & -0.13 & 0.29 & 0.04 & 0.11 \\
\hline & $36 \mathrm{~d}$ & -0.07 & -0.09 & 0.00 & $0.10^{*}$ & 0.00 & 0.00 & 0.00 & 0.16 & -0.04 & 0.10 & -0.01 \\
\hline & $43 \mathrm{~d}$ & 0.00 & -0.16 & 0.18 & 0.00 & $0.40^{+}$ & -0.21 & 0.00 & 0.06 & -0.10 & 0.29 & -0.15 \\
\hline \multirow[t]{5}{*}{$\mathrm{P}_{6}$} & $15 \mathrm{~d}$ & 0.00 & $0.27^{*}$ & 0.04 & 0.00 & $-0.31^{+}$ & $0.39^{*}$ & 0.00 & -0.05 & 0.03 & -0.08 & 0.46 \\
\hline & $22 \mathrm{~d}$ & 0.00 & $0.63^{*}$ & $-0.46^{*}$ & 0.00 & 0.31 & -0.10 & 0.00 & $-0.69^{*}$ & $0.64^{* *}$ & 0.24 & 0.08 \\
\hline & $29 \mathrm{~d}$ & 0.00 & -0.28 & 0.28 & 0.00 & -0.15 & 0.54 & 0.00 & 0.15 & 0.06 & -0.28 & 0.88 \\
\hline & $36 \mathrm{~d}$ & $0.17^{+}$ & $0.27^{+}$ & 0.03 & -0.02 & 0.00 & 0.00 & 0.00 & $-0.26^{*}$ & $0.21^{*}$ & 0.16 & 0.32 \\
\hline & $43 \mathrm{~d}$ & 0.00 & 0.05 & 0.07 & 0.00 & -0.03 & 0.02 & $0.15^{+}$ & 0.24 & 0.00 & 0.40 & 0.24 \\
\hline \multirow[t]{5}{*}{$\mathrm{P}_{7}$} & $15 \mathrm{~d}$ & 0.00 & $0.18^{+}$ & -0.27 & 0.00 & 0.28 & 0.03 & 0.00 & -0.10 & 0.09 & 0.35 & -0.15 \\
\hline & $22 \mathrm{~d}$ & 0.00 & $0.72^{*}$ & -0.54 & 0.00 & $0.51^{*}$ & $-0.38^{+}$ & 0.00 & $-0.86^{* *}$ & $0.73^{* *}$ & 0.37 & -0.19 \\
\hline & $29 \mathrm{~d}$ & 0.00 & -0.65 & 0.39 & 0.00 & $-0.29^{*}$ & 0.04 & 0.00 & 0.44 & -0.43 & -0.50 & 0.00 \\
\hline & $36 \mathrm{~d}$ & -0.17 & $-0.33^{+}$ & 0.13 & -0.22 & 0.00 & 0.00 & 0.00 & $0.32^{*}$ & $-0.27^{*}$ & -0.40 & -0.54 \\
\hline & $43 \mathrm{~d}$ & 0.00 & -0.07 & 0.00 & 0.00 & 0.08 & -0.12 & -0.04 & -0.12 & 0.03 & -0.14 & -0.14 \\
\hline \multirow[t]{5}{*}{$\mathrm{P}_{8}$} & $15 \mathrm{~d}$ & 0.00 & $0.37^{*}$ & $-0.18^{+}$ & 0.00 & 0.23 & -0.17 & 0.00 & $-0.35^{* *}$ & 0.23 & 0.25 & -0.12 \\
\hline & $22 \mathrm{~d}$ & 0.00 & 0.02 & -0.06 & 0.00 & -0.04 & 0.04 & 0.00 & -0.06 & 0.01 & -0.08 & 0.00 \\
\hline & $29 \mathrm{~d}$ & 0.00 & -0.16 & 0.27 & 0.00 & 0.07 & 0.12 & 0.00 & 0.11 & -0.30 & 0.02 & 0.08 \\
\hline & $36 \mathrm{~d}$ & $-0.17^{*}$ & 0.02 & $-0.24^{+}$ & 0.28 & 0.00 & 0.00 & 0.00 & -0.12 & 0.04 & 0.01 & -0.09 \\
\hline & $43 \mathrm{~d}$ & 0.00 & $-0.14^{*}$ & 0.00 & 0.00 & $-0.26^{*}$ & $0.28^{*}$ & -0.07 & 0.07 & $-0.20^{+}$ & -0.40 & 0.01 \\
\hline \multirow[t]{5}{*}{$\mathrm{P}_{9}$} & $15 \mathrm{~d}$ & 0.00 & 0.29 & -0.30 & 0.00 & $-0.94^{*}$ & 1.17 & 0.00 & -0.16 & 0.11 & -0.80 & 0.98 \\
\hline & $22 \mathrm{~d}$ & 0.00 & -0.07 & -0.01 & 0.00 & -0.14 & -0.22 & 0.00 & 0.09 & -0.09 & -0.11 & -0.32 \\
\hline & $29 \mathrm{~d}$ & 0.00 & -0.21 & 0.20 & 0.00 & $-0.23^{*}$ & $0.69^{*}$ & 0.00 & 0.11 & -0.32 & -0.34 & 0.57 \\
\hline & $36 \mathrm{~d}$ & 0.04 & 0.07 & 0.00 & -0.02 & 0.00 & 0.00 & 0.00 & $-0.21^{*}$ & 0.12 & -0.13 & 0.15 \\
\hline & $43 \mathrm{~d}$ & 0.00 & $0.24^{+}$ & $-0.24^{*}$ & 0.00 & -0.18 & 0.10 & -0.13 & $-0.35^{+}$ & 0.14 & -0.41 & -0.13 \\
\hline
\end{tabular}

$+{ }^{*}$ 和 ${ }^{* *}$ 分别为达到 0.10、0.05 和 0.01 显著水平。Gt: 总遗传效应值, 1 和 2 分别表示 2006 年和 2007 年。

$+{ }^{*}$ and ${ }^{* *}$ : significant at $0.10,0.05$, and 0.01 probability levels, respectively. $\mathrm{P}_{1}$ : Youcai $601 ; \mathrm{P}_{2}$ : Shuang $20-4 ; \mathrm{P}_{3}$ :Huanshuang $3 ; \mathrm{P}_{4}$ : Gaoyou 605; $\mathrm{P}_{5}$ : Zhongyou 821; $\mathrm{P}_{6}$ : Eyouchangjia; $\mathrm{P}_{7}$ : Zhong R-888; $\mathrm{P}_{8}$ : Tower; $\mathrm{P}_{9}$ : Zheshuang 72.

$A o$ : embryo additive main effect; $A o E$ : embryo additive interaction effect; $C$ : cytoplasm main effect; $C E$ : cytoplasmic interaction effect; $A m$ : maternal additive main effect; $A m E$ : maternal additive interaction effect. Gt: total genetic effect, 1 and 2 referred to 2006 and 2007 , respectively. 
相对稳定; 而有些亲本受环境影响较大, 年份间出现较大的 波动。就总体而言, 鄂油长荚 $\left(\mathrm{P}_{6}\right)$ 和高油 $605\left(\mathrm{P}_{4}\right)$ 在油菜籽发 育过程中的遗传效应相对较为稳定, 不同年份间提高后代 赖氨酸含量的正值较多, 有利于稳定地增加杂种后代油菜 籽的赖氨酸含量; 其中以鄂油长荚更为理想(2007 年油菜 籽各发育时段的正向预测值分别为 $0.46 \% 、 0.08 \% 、 0.88 \%$ 、 $0.32 \%$ 和 $0.24 \%$ ), 不同发育时期均能增加后代的赖氨酸含 量。而中 R-888 $\left(\mathrm{P}_{7}\right)$ 和浙双 72 $\left(\mathrm{P}_{9}\right)$ 等亲本则表现为以负值为主, 其遗传效应预测值不利于增加杂种后代的赖氨酸含量。其他 亲本受环境的影响较大, 不同发育时期表现出的遗传效应 在年份间的稳定性不够。

\section{3 讨论}

早期的发育遗传研究一般以经典的数理统计方法为 基础, 采用世代平均数计算遗传效应值及方差; 或采用简 单的遗传模型估算各遗传方差和协方差分量, 分析控制 数量性状的基因表达在不同发育阶段中的变化 ${ }^{[14-15]}$ 。但这 些研究未能发现不同遗传体系基因的表达特性及其油菜 籽特定发育阶段品质性状形成的动态遗传机理。理想的数 量性状发育遗传模型和统计分析方法应能反映研究性状 的遗传复杂性和发育过程中的动态本质。 $\mathrm{Zhu}^{[13]}$ 首次提出 的条件遗传分析方法可用于发育数量遗传的分析, 估算 出特定发育阶段基因表达的遗传效应, 较好地克服了传 统数量遗传方法未能准确检测特定发育时期基因表达的 不足, 可以用于深入研究不同发育时段多遗传体系影响 油菜籽品质性状表达的遗传机理。本研究应用条件与非条 件遗传分析方法, 获得了油菜籽饼粕赖氨酸含量性状在 2 个任意发育时段之间的遗传效应净增量或从开花到某一 发育时段的累加效应, 而且还能将累加效应或净效应分 解为胚加性效应、胚显性效应、细胞质效应、母体加性效 应、母体显性效应及其相应的环境互作效应, 明确各遗传 分量对油菜籽饼粕赖氨酸含量性状表现的相对贡献。因此, 油菜籽饼粕赖氨酸含量涉及众多基因的调控和相互作用, 其遗传效应的表达与发育时期和环境条件有着密切关系, 存在着基因表达的发育阶段性以及基因表达类型和程度 上的差异。品质性状最终表型值决定于整个油菜籽发育过 程中众多基因的表达、调控和相互作用，同时也还会受到 不同发育时段和基因与环境互作以及环境条件的影响。环 境条件的不同使得基因在不同发育时期间所表达的遗传 效应有着明显差异, 对该品质性状的表现产生较大影响。 油菜籽饼粕赖氨酸含量在发育中的动态是一个调整的、互 作的、连续的、可遗传的、渐成的基因表达过程。

油菜属于双子叶作物, 其二倍体胚(子叶)的营养物 质由不同世代的二倍体母体植株所提供, 故油菜籽的一 些数量性状会同时受到胚(子叶)和/或母体植株核基因的 控制; 同时细胞质基因也可能通过控制叶绿体(或线粒体) 的合成而影响光合作用(或呼吸作用), 间接影响油菜籽品 质性状的表现 ${ }^{[16-17]}$ 。油菜籽饼粕赖氨酸含量等种子品质性
状属于数量性状, 众多基因在特定遗传背景和环境条件 下的表达可以影响该性状在不同发育阶段中的表现。本研 究结果表明，油菜籽饼粕赖氨酸含量在不同的发育时期 同时受到胚、细胞质和母体植株等遗传体系基因主效应以 及环境互作效应的影响, 基因的表达与油菜籽的发育进 程关系密切, 具有时间序列特征。因此, 发育遗传机理的 研究有助于明确各发育时期油菜籽饼粕赖氨酸含量等品 质性状的多种遗传效应，有利于提高油菜品种选育的效 率。不同发育时期油菜籽饼粕赖氨酸含量性状的遗传分析 表明，二倍体胚和二倍体母体植株基因的加性互作效应 和显性互作效应以及细胞质效应可以明显影响各个发育 时期油菜籽垪粕的赖氨酸含量, 且是以母体植株加性互 作效应为主, 对油菜籽饼粕赖氨酸含量的选择可望取得 较好的效果。条件方差分量分析结果则表明了胚、细胞质 和母体植株等遗传体系的基因在油菜籽各个发育时期中 均有新的表达, 且以油菜籽发育前期和中期为主, 其中花 后 23 29 d 表达的基因效应尤为明显，说明花后 23 29 d 是该性状基因表达最为活跃的时期，各种基因效应对油 菜籽饼粕赖氨酸含量具有最为重要的作用。同时, 这些结 果有效地揭示不同环境条件下油菜籽饼粕赖氨酸含量在 发育过程中, 数量基因的动态表达过程以及特定发育时 期数量基因所产生净遗传效应，为不同发育时期有关油 菜籽饼粕赖氨酸含量性状的 QTL 定位和进行相应的分子 标记辅助选择提供了一定的理论依据。

本研究还发现赖氨酸含量具有较高的狭义遗传率, 与母体植株有关的遗传率明显大于胚遗传率, 说明在育 种过程中可以根据母体植株上油菜籽饼粕赖氨酸含量的 总体表现进行选择。在亲本的选择上, 由于采用传统的方 法, 仅分析成熟时段油菜籽饼粕赖氨酸的最终遗传表现, 很难揭示整个发育过程中各个时期基因的遗传效应差异。 因此，深入分析不同亲本在各个发育时段的作用和遗传 效应差异, 对提高油菜籽饼粕赖氨酸含量的育种效率有 重要指导作用。本试验中鄂油长荚和高油 605 的遗传表现 在不同环境下较为稳定, 多数发育时期是以能够增加饼 粕赖氨酸含量的正值为主, 受环境影响相对较小, 宜作为 育种改良的亲本，有利于提高杂种后代油菜籽饼粕的赖 氨酸含量。

\section{References}

[1] Xiong Z-Y(熊志勇), Xia F-J(夏伏建), Lu S-G(陆师国). The protein classification of fine rapeseed cake. J Wuhan Bot Res (武汉 植物学研究), 2001, 19(3): 259-261 (in Chinese)

[2] Shi C H, Xue J M, Yu Y G, Yang X E, Zhu J. Analysis of genetic effects on nutrient traits in indica rice. Theor Appl Genet, 1996, 92: 1099-1102

[3] Shi C H, Zhu J, Wu J G, Yang X E, Yu Y G. Analysis of embryo, endosperm, cytoplasmic and maternal effects for heterosis of protein and lysine content in indica hybrid rice. Plant Breed, 1999, 118: $574-576$ 
[4] Yan X-F(间新甫), Xu S-Y(徐绍英), Li W-F(李卫芬), Chen $\mathrm{H}-\mathrm{J}($ (陈浩军), Zhu J(朱军). Analysis of seed and maternal genetic effects on the contents of seven essential amino acids in two-rowed barley. Sci Agric Sin (中国农业科学), 1997, 30(2): 34-41 (in Chinese with English abstract)

[5] Wu J G, Shi C H, Zhang X M, Katsura T. Genetic and genotype × environment interaction effects for the content of seven essential amino acids in indica rice. $J$ Genet, 2004, 83: 171-178

[6] Wu J G, Shi C H, Zhang X M, Katsura T. Genetic analysis of non-essential amino acid contents in rice (Oryza sativa L.) across environments. Hereditas, 2004, 141: 128-134

[7] Wu J G, Shi C H, Zhang H Z. Genetic analysis of embryo, cytoplasmic, and maternal effects and their environment interactions for protein content in Brassica napus L. Aust J Agric Res, 2005, 56: 69-73

[8] Ren Y-L(任玉玲), Shi C-H(石春海)，Wu J-G(吴建国), Zhang $\mathrm{H}-\mathrm{Z}$ (张海珍). Analysis of embryo, cytoplasmic and maternal effects on three amino acid traits in rapeseed. $J$ Zhejiang Univ (Agric \& Life Sci) (浙江大学学报·农业与生物技术版), 2005, 31 (1): 41-46 (in Chinese with English abstract)

[9] Variath M T, Wu J G, Li Y X, Chen G L, Shi C H. Genetic analysis for oil and protein contents of rapeseed (Brassica napus L.) at different developmental times. Euphytica, 2009, 166: 145-153

[10] Wu J-G(吴建国), Shi C-H(石春海), Zhang H-Z(张海珍). Study on developing calibration models of fat acid composition in intact spectroscopy and spectral analysis rapeseed by near infrared reflectance spectroscopy. Spectr Spectr Anal (光谱学与光谱分析), 2006, 26(2): 259-262 (in Chinese with English abstract)

[11] Wu J G, Shi C H, Zhang X M. Estimating the amino acid composition in the milled rice powder by near-infrared reflectance spectroscopy. Field Crops Res, 2002, 75: 1-7

[12] Zhu J, Weir B S. Analysis of cytoplasmic and maternal effects: I. A genetic model for diploid plant seeds and animals. Theor Appl Genet, 1994, 89: 153-159

[13] Zhu J. Analysis of conditional genetic effects and variance components in developmental genetics. Genetics, 1995, 141: 1633-1639

[14] Griffing B. Concept of general and specific combining ability in relation to diallel crossing system. Aust J Biol Sci, 1956, 9: 463-493

[15] Hayman B I. The theory and analysis of diallel cross. Genetics, 1954, 139: 799-809

[16] Shi C H, Wu J G, Wu P. Developmental behavior of gene expression for brown rice thickness under different environments. Genesis, 2002, 33: 185-190

[17] Shi C H, Zhang H Z, Wu J G, Li C T, Ren Y L. Genetic and genotype $\times$ environment interaction effects analysis for erucic acid content in rapeseed (Brassica napus L.). Euphytica, 2003, 130: 249-254 